\title{
Active Brain Model Based on Neuromorphic Networks with the Implementation of Cognitive Functions
}

\author{
Andrey Molyakov* \\ Institute of information technologies and cybersecurity, Russia \\ *Corresponding author: Andrey Molyakov, Institute of information technologies and cybersecurity, Russian State \\ University for the Humanities, Russia
}

\section{ARTICLE INFO}

Received: 幽 July 03, 2020

Published: 幽 July 13, 2020

Citation: Andrey Molyakov. Active Brain Model Based on Neuromorphic Networks with the Implementation of Cognitive Functions. Biomed J Sci \& Tech Res 28(5)2020. BJSTR. MS.ID.004712.
ABSTRACT

Deep learning neural networks will help not only to decipher the principles of the human brain, but also to establish a permanent connection with it. This idea is being developed now in the laboratory of neurocomputer interfaces (Russian State University for the Humanities). Scientists want to load as many electroencephalograms (EEGs) into the computer as possible and set the task of neural networks to detect differences in them with simple commands - for example, turn right / left. If the connection between artificial and human intelligences is established, people will be able to operate thousands of times with large volumes of information, without violating the natural manifestations of feelings, emotions and consciousness caused by human nature.

Keywords: Neuronal model; Quantum Mechanics; Consciousness; Wave Theory; Biocomputer

\section{Introduction}

American scientists from the University of California at Los Angeles together with their colleagues from the National Institute of Materials Science of Japan (NIMS) created a neuromorphic network, in fact, an active model of the brain. The device is a tangled "ball" of the thinnest silver nanowires with an average diameter of 360 nanometers, coated with an insulating polymer with a thickness of only 1 nanometer. The size of the entire device is 10 square millimeters. Randomly assembled on a silicon wafer, the nanowires formed highly organized structures that are surprisingly similar to those that form the neocortex - the part of the brain involved in higher functions such as language, perception, and cognition. At the intersection of nanowires, due to the migration of silver atoms through the polymer coating, compounds formed similar to neural synapses, due to which brain cells connect and communicate. In total, the system created about 10 million such compounds. Each time scientists applied voltage to the two ends of the neuromorphic network, she "tried" to find the optimal, most electrically effective paths, that is, the "synaptic elements" worked together to optimize the current transfer.

Self-organizing dynamic processes arose. Measuring the parameters of these processes - the time of the current path, delays and deactivation - the authors found that they are very similar to the parameters of the processes occurring in the brain $[1,2]$. Using neuromorphic deposition, scientists were able to reproduce electrical characteristics similar to those associated with human functions of a high-order brain, such as remembering, learning, forgetting, wakefulness and sleep. In some cases, when the current flowed through the network, the bonds between the nanowires were maintained for one minute, which was reminiscent of the learning and memorization process, in other cases, the connection was abruptly cut off after the charge ended, simulating the forgetting process. In other experiments, scientists found that with low energy consumption, the device exhibits behaviors consistent with the state of sleep. With more power, the network behaved like an awake brain $[3,4]$. Organoids of the cerebral cortex were grown by laboratory staff on special chips that could record activity to obtain more accurate data without the need for additional complex work. The risk of material damage that was unavoidable during measurements was also minimized.

\section{Recognition and Specific Language}

The practical implementation of this approach is to put a single task before the brain and the artificial neural network (ANN): to 
adapt to each other in order to form and accept teams that are understandable for both sides. For ANNs, this is a typical pattern recognition task. Only the images are not car numbers and not the faces of criminals, but EEG indicators, which reflect the intentions of a person. The novelty of the project is that the results of the work of the neural network will in some way inform the brain, which in turn will begin to rearrange its activity in order to become more understandable for the computer. As a result, the unbelievable can happen - between the brain and the computer without the intervention of researchers a specific language of communication will be developed. The experiment is as follows. At the input of the neural network will supply the EEG recorded from the subjects. For example, when they represent the movement of their left or right hand. The task of the ANN is to find the differences. Initially, they may not even exist, but since the brain and the neural network are interested in getting the result, the brain will sort out the possibilities (change the EEG) to become clear to the ANN, and it will restructure its structure to evaluate these efforts.

It is important that during training, as the ANN is trained, for the first time in the world itself will become the object of fundamental neurophysiological research. After all, not being biased by "knowledge" about the nature and phenomenology of EEG, about different alpha-beta rhythms, trained to distinguish between brain states, the neural network will contain the signs of EEG found by itself, by which it draws the correct conclusions, that is, for example, distinguish a right turn from a left turn. This will be the most interesting for neurophysiologists, who in this case will come "to the ready." For the first time, important signs of EEG will not be found in laboratory analyzes, but in the process of interaction on the line "brain - artificial intelligence". "There will be no reason for researchers to" manually "choose EEG indicators taken from textbooks, for example, the notorious alpha rhythm, in order to build teams from the brain to executive systems on them," - explained Alexander Kaplan. Now this choice seems unnatural, because the same alpha rhythm in the EEG probably reflects some important brain process and making this process work as brain commands is the same as using pressure in a steam boiler to transmit a message in Morse code. And if you allow the brain to independently "negotiate" with the neural network exactly what signs of the EEG can be used as commands, then a real communication channel will be obtained between the two intellects.

With the successful implementation of the idea of establishing a direct channel of communication between the ANN and the brain, completely new possibilities will open before a person: for example, it will be possible to rely on the control of processor cells as additional memory and to accommodate the most difficult search operations for the brain in them. Such an experiment can bring large-scale results. Artificial neural networks offer extraordinary opportunities for neuroscience. If in medicine they are able to diagnose diseases, then in the activity of the brain they are trained to recognize internal speech, images, movements, even intentions to them. However, this is only one side of the story unfolding today - to use the learning ability of an artificial network to recognize intentions from the natural. But the natural neural network is also capable of learning. Professor Kaplan's original idea, which is now being implemented in his laboratory, is to close these two learning networks in a ring with constant feedback to each other. I have not seen works with such a plan.

\section{Conclusion}

It should be noted that this idea has a great future. Of particular interest is the idea of the interaction of artificial intelligence with brain activity, because such an intellect will be able to choose the most suitable visual (and, potentially, auditory, and tactile) stimuli for the interface. The quality of reading thoughts will undoubtedly improve. Such neural interfaces will be very useful in medicine to restore functions in patients with neurological lesions and to accelerate their rehabilitation. If Russian scientists manage to establish a stable communication channel, artificial intelligence will not be a competitor, but an assistant to the human brain, which will provide its creative potential with enormous memory and speed. Not only new fundamental discoveries await the person along this path, but also a significant expansion of his capabilities. He will be able to instantly connect to different language databases, which will give him the opportunity to speak any languages without long training, operate thousands of times with large volumes of information. In this case, the person will remain human. The phantasmagoric idea of enslaving him with artificial intelligence will forever be a thing of the past.

\section{References}

1. Turin L, Skoulakis EMC, Horsfield AP (2014) Electron spin changes during general anesthesia in Drosophila. Proc Nat Acad Sci USA 111(34): E3524-E3533.

2. Koch C (2004) The Quest for Consciousness: A Neurobiological Approach (Roberts \& Company).

3. Tononi G (2004) An information integration theory of consciousness. BMC Neuroscience 5: 42.

4. Tegmark M (2015) Consciousness as a state of matter. Chaos, Soli-tons \& Fractals 76: 238-270. 
ISSN: 2574-1241

DOI: 10.26717/BJSTR.2020.28.004712

Andrey Molyakov. Biomed J Sci \& Tech Res

(C) (P) This work is licensed under Creative

Submission Link: https://biomedres.us/submit-manuscript.php

\begin{tabular}{ll} 
BIOMEDICAL & Assets of Publishing with us \\
RESEARCHES & - Global archiving of articles \\
& - Immediate, unrestricted online access \\
\hline
\end{tabular}

